
\title{
$\begin{array}{ll}\text { Research Square } & \text { They should not be considered conclusive, used to inform clinical practice, } \\ \text { or referenced by the media as validated information. }\end{array}$ \\ RelB Upregulates PD-L1 and Exacerbates Prostate Cancer Immune Evasion
}

\section{Yanyan Zhang}

Nanjing Medical University

\section{Shuyi Zhu}

Nanjing Medical University

\section{Yuanyuan Du}

Nanjing Medical University

\section{Fan Xu}

Nanjing Medical University

\section{Wenbo Sun}

Nanjing Medical University

\section{Zhi Xu}

Nanjing Medical University

\section{Xiumei Wang}

Nanjing Medical University

\section{Peipei Qian}

Nanjing Medical University

Qin Zhang

Nanjing Medical University

Jifeng Feng

Nanjing Medical University

Yong Xu ( $\nabla$ yxu4696@njmu.edu.cn )

Nanjing Medical University https://orcid.org/0000-0002-8361-9970

\section{Research Article}

Keywords: PD-L1, RelB, T-cell immunity, immune evasion, prostate cancer

Posted Date: September 8th, 2021

DOI: https://doi.org/10.21203/rs.3.rs-861355/v1

License: (c) (1) This work is licensed under a Creative Commons Attribution 4.0 International License. Read Full License 


\section{Abstract}

\section{Background}

Interaction between programmed death receptor (PD-1) and its ligand (PD-L1) is essential for suppressing activated T-lymphocytes. However, the precise mechanisms underlying PD-L1 overexpression in tumors remain to be fully elucidated. Here, we describe that RelB participates in immune evasion of prostate cancer (PCa) via cis/trans transcriptional upregulation of PD-L1.

\section{Methods}

Based on transcriptome results, RelB was manipulated in multiple human and murine PCa cell lines. The activated $\mathrm{CD}^{+}$and $\mathrm{CD}^{+}{ }^{+} \mathrm{T}$ cells were co-cultured with PCa cells with different levels of RelB to examine the effect of tumorous RelB on T-cell immunity. Male mice were injected with murine PCa cells to validate the effect of RelB on the PD-1/PD-L1-mediated immune checkpoint using both tumor growing and metastatic experimental models.

\section{Results}

PD-L1 uniquely expresses at a high level in PCa with high constitutive RelB and correlates to the patients' Gleason scores. Indeed, the ligh level of PD-L1 is associated with RelB nuclear translocatiob in ARnegative aggressive PCa cells. The silence of RelB in advanced PCa cells resulted in reducing PD-L1 expression and enhancing the susceptibility of PCa cells to the T-cell immune response in vitro and in vivo. Mechanistically, a proximal NF-kB enhancer element was identified in the core promoter region of the human CD274 gene, which is responsible for RelB-mediated PD-L1 transcriptional activation. The finding provides an informative insight into immune checkpoint blockade by administering RelB within the tumor microenvironment.

\section{Conclusions}

This study deciphers the molecular mechanism by which tumorous RelB contributes to immune evasion by inhibiting T-cell immunity via the amplification of PD-L1/PD-1-mediated immune checkpoint.

\section{Background}

Prostate cancer ( $\mathrm{PCa}$ ) is the most common malignant tumor and the leading cause of cancer death in men, with an estimated 1276000 new cases and 359000 deaths worldwide in 2018 [1]. Owing to the improved early diagnosis and the advanced therapeutic strategies, the mortalities of PCa have been appreciably decreased. Unfortunately, a part of PCa patients eventually developed more aggressive malignant forms resistant to traditional radiotherapy and chemotherapy, leading to poor prognosis [2]. The emerging evidence demonstrated that the enhancement of immune response is an effective therapeutic option for improving the survival of cancer patients [3]. Indeed, tumor-induced immunosuppression plays a pivotal role in tumor evasion from the host immune surveillance, which 
provides a complementary approach to treat tumors using immune-checkpoint inhibitors [4]. Accordingly, the mounting studies have focused on blocking immune checkpoints, such as targeting CTLA-4 and PD1/PD-L1 axis, which has been shown to restore or strengthen T-cell antitumor immunity $[5,6]$. Especially, PD-1/PD-L1 is widely recognized as the most outstanding immune checkpoint target based on its therapeutic validation in prospective clinical outcomes, including subsets of solid tumors [7].

PD-1, an important T-cell co-inhibitory receptor, expresses on the surface of antigen-stimulated T cells and plays a fundamental role in suppressing the adaptive immune responses and promoting self-tolerance against T-cell inflammatory activity. The activation of PD-1 prevents autoimmune diseases and impedes tumor suppression due to immuno-compromise [8]. PD-1 has two known ligands, PD-L1 and PD-L2, of which PD-L1 is the dominant inhibitory ligand associated with immunotherapeutic responses within the tumor microenvironment [9]. As a critical checkpoint molecule in the regulation of immune response, PD-1 expresses in dendritic cells, T/B cells, tumor-associated macrophages, and myeloid-derived suppressor cells [10]. On the other hand, PD-L1 highly expresses in a variety of malignant cancer cells, leading to tumor immune evasion by interaction with PD-1 [11]. Since PD-L1/PD-1 interaction is essential for the induction of T-cell apoptosis and immune checkpoint response, targeting the PD-1/PD-L1 axis is thought to be a feasible approach for treating aggressive tumors [12].

The transcription of PD-L1 is regulated by inflammatory signaling pathways. Particularly, IFN- $y$, a proinflammatory cytokine, is recognized as the most prominent inducer of PD-L1 [13]. Additionally, several other cytokines are also able to upregulate PD-L1, including TNF-a, IL-13, IL-4, IL-10, and IL-17 [14]. Multiple transcription factors involved in the JAK/STAT, RAS/MAPK, and PTEN-PI3K/AKT pathways participate in the regulation of PD-L1, such as STAT1, STAT3, IRF1, IRF3, HIF-1a, MYC, JUN, BRD4, and NF-KB [15]. Since NF-KB plays a substantial role in inflammatory and immune responses [16], NF-KB is considered to influence PD-L1 expression. RelA (p65) has been reported to upregulate PD-L1 expression in lung cancer cells in response to TNF-a stimulation [17].

NF-KB is well-documented to play an essential role in cancer progression and therapeutic resistance [18]. In general, the activation of NF-KB is mediated by two major pathways, i. e., canonical pathway and noncanonical pathway. The canonical pathway is quickly responsive to many exogenous stimulants and leads to the nuclear translocation of p50:RelA dimer followed by IкB phosphorylation and degradation [19]. While the noncanonical pathway is gradually but persistently activated by processing p100:RelB into p52:RelB dimer [20]. In addition to the canonical pathway that is widely recognized to be critical for regulating inflammatory response, the noncanonical pathway is thought to be a key regulator for the immune response [21]. Nevertheless, in contrast to the well-studied canonical pathway in cancer development, the role of the noncanonical pathway in cancer remains to be fully elucidated.

RelB has been originally identified in B-cells and the RelB-activated noncanonical NF-KB pathway has been shown to respond to antigen presentation by DCs, which is associated with inflammation and excessive immune cell infiltration [22-24]. RelB has been implicated in cancer progression, particularly in sex hormone-related cancers including PCa, breast cancer and endometrial cancer [25-27]. We have 
recently shown that the high constitutive level of RelB is correlated to PCa radioresistance [28]. The present study further demonstrates a cis/trans transcriptional regulatory mechanism by which RelB upregulates PD-L1 in PCa cells. Accordingly, the repression of tumor-derived RelB can promote the T-cell immune response by downregulating PD-L1.

\section{Methods}

\section{PCa patients}

Nanjing Medical University affiliated Cancer Hospital (Nanjing, China) has collected fresh tumor tissues from newly diagnosed PCa patients before chemotherapy or radiotherapy. The Ethics Committee of Nanjing Medical University has approved the study protocol with written informed consent forms obtained from patients enrolled in this study. From Gleason score 5 to 9, each group containing 8-10 cases were selected to examine the correlation between RelB and PD-L1 expression using immunohistochemistry (IHC) with monoclonal antibodies agranst human RelB and PD-L1 (Cell Signaling Tech., USA ). Briefly, the tissues were fixed in paraffin-embedded slides and then dewaxed by xylene. After washing with ethanol, the tissue slides were further rehydrated by rinsing with $\mathrm{dH}_{2} \mathrm{O}$. For $\mathrm{IHC}$, the tissue slides were soaked in $5 \%$ BSA buffer for $1 \mathrm{~h}$ and then incubated with 400x diluted primary antibodies within $5 \% \mathrm{BSA}$ buffer at $4^{\circ} \mathrm{C}$ overnight. After washing with PBS, the tissue slides were incubated with biotinylated secondary antibody at room temperature for $30 \mathrm{~min}$. After washing with BPS, a DAB Substrate Kit (Cell Signaling Tech., USA) was used to observe immunostaining images under a microscope. The intensity of IHC staining was scored as negative (score 0), weak (1), medium (2), and strong (3). Total cell positivity was scored as the percentage of positive cells, including no positive cell $(0),<25 \%$ (1), 25-50\% (2), 50-75\% (3), and $>75 \%$ (4), respectively. The "H-score" was calculated using " $\sum \mathrm{pi}(\mathrm{i}+1)$ " for all slides, in which "pi" represented the percentage of positive and " $\mathrm{i}$ " represented the staining intensity.

\section{Mice}

Animal experiments were performed according to the Institutional Animal Care and Use approved by the Research Committee of Nanjing Medical University (No. IACUC-1901031). For the mouse xenograft PCa tumor growth experiment, the five-week-old male C57BL/6 mice (Beijing Vital River Laboratory Animal Technology Co., Ltd., China) were randomly divided into three groups. The mice were subcutaneously injected $2.5 \times 10^{5}$ RM- 1 cells with different levels of RelB and PD-L1 into the left flank. The formed tumors were measured using digital calipers every other day and tumor volume was calculated using a standard formula ( $V=0.52 \times \mathrm{AB}^{2}, A$ and $B$ represent the diagonal tumor lengths). $7,14,21$ days after the cellinjection, $\mathrm{CD} 4^{+}$and $\mathrm{CD} 8^{+} \mathrm{T}$ cells were isolated from mice blood samples and analyzed by flow cytometry. The mice were finally executed and tumor tissues were excised for immunoblots and IHC. For xenograft PCa tumor metastatic experiment, $5 \times 10^{4} \mathrm{RM}-1$ cells were injected into mice through the tail vein. The mice were euthanized four weeks later to remove lung tumor tissues and analyzed by $\mathrm{IHC}$ and 
immunoblots. Additionally, $\mathrm{CD} 4^{+}$and $\mathrm{CD} 8^{+} \mathrm{T}$ cells isolated from mice spleen tissues were analyzed by flow cytometry.

\section{T-cell preparation}

Whole blood samples were obtained from healthy donors according to the institutional guidelines with informed consent. Peripheral blood mononuclear cells (PBMC) were isolated by density centrifugation using Ficoll gradient separation. The cells were washed three times with PBS and suspended in RPMI 1640 media (Gibco, USA). In addition, mouse T cells were isolated from peripheral blood samples and spleen tissues derived from male C57BL/ 6 mice. The pellets were lysed within a RBC lysis buffer for 10 min to remove red blood cells and the tissues were grinded and then filtered to remove tissue debris. The cells were cultured in a T-cell preconditional RPMI 1640 media supplemented with 1000x mercaptoethanol (Gibco), 100x penicillin-streptomycin (Gibco), 100X glutamine-MAX (Gibco) and 10\% heat inactivated FBS (Gibco). The single cells were collected by centrifugation and T-cell was stimulated in media containing $2 \mu \mathrm{g} / \mathrm{ml}$ anti-CD3, $3 \mu \mathrm{g} / \mathrm{ml}$ anti-CD28 and $200 \mathrm{U} / \mathrm{ml} \mathrm{IL-2} \mathrm{(Biolegend,} \mathrm{Inc.,} \mathrm{USA)} \mathrm{for} 24 \mathrm{~h}$ prior to co-culture with PCa cells.

The cultured T-cell was analyzed by flow cytometry before and after co-culture with PCa cells. APCconjugated anti-human CD4/mouse antibody (Biolegend) and PE-conjugated anti-human CD8/mouse antibody (BioLegend) were used to stain CD4 or CD8 cells. The labeled cells were analyzed by a BD FACSCalibur flow cytometer (BD Sciences, USA). In addition, to analyze T-cell proliferation, the $T$ cells were labeled with $5 \mu \mathrm{M}$ CFSE (Biolegend) for $20 \mathrm{~min}$ at $37^{\circ} \mathrm{C}$ in darkness. The cell staining was blocked by adding cell culture media containing $10 \%$ FBS. Flow cytometry data were further analyzed with FlowJo software and Modfit software.

\section{RelB manipulation in PCa cells}

Human PCa cell lines (PC-3, DU-145) and mouse PCa cell lines (RM-1) were purchased from the American Type Culture Collection (ATCC, USA). The cell lines were cultured in the recommended media containing $10 \%$ FBS and $1 \%$ penicillin/streptomycin. The cells were treated with IFN-y (Novus, USA) to induce PD-L1 expression. RelB was silenced in PC-3 and DU-145 cells by transfected a plasmid-carried a shRNA duplex targeting RelB (RiboBio Co., Ltd., China) and stable cell clones were selected using G418 (Invitrogen, USA). Additionally, RelB was knocked out (RelB-KO) in RM-1 cells using a CRISPR/Cas9-based gene edition system. Briefly, RM-1 cells were transfected with a Cas9-single guide RNA (sgRNA) expression plasmid targeting RelB (sgRNA, 5'-GACGAATACATTAAGGAGAA-3') and followed by puromycin selection. Furthermore, PD-L1 CDNA was cloned in pcDNA plasmid and then transfected into the RelB-KO RM-1 cells and followed by hygromycin B (Invitrogen) selection to generate a stable cell line.

\section{Immune cytotoxicity}

The effect of activated T cells on the survival of PCa cells was determined using cell counting and clonogenic assay, respectively. After co-cultured with activated T cells at a 1:10 ratio for 3 days, PCa cells were seeded into 96 -well plates at a density of $10^{3}$ cells/well and then cultured for 2 days. The cells were 
treated with a CCK-8 reagent (Dojindo Mol. Tech., Japan), and cell viability was measured as the optical density at $450 \mathrm{~nm}$. For the clonogenic assay, PCa cells were treated with an anti-PD-L1 mAb (abcam, USA) prior to co-culture with activated T cells. Thereafter, 100-200 PCa cells were plated in 6-well plates and continuously cultured allowing colony formation. After washing with 1x PBS twice, the colonies were stained with $1 \%$ crystal violet dye for $30 \mathrm{~min}$ to form visible cell clones. The cell surviving fractions were calculated based on the ratio of the number of colonies formed to the number of cells efficiently plated.

\section{RNA-seq}

The RNA sequencing libraries were conducted by Vazyme Biotech Co., Ltd (Nanjing, China) using a VAHTS mRNA-seq v2 Library Prep Kit for Illumina. The cDNA libraries were sequenced on an Illumina Hiseq $\mathrm{X}$ Ten platform with $150 \mathrm{bp}$ paired-end module. The raw reads were filtered by removing reads containing adapter, ploy-N and low-quality reads for subsequent analysis. Cuffdiff (v1.3.0) was used to calculate FPKMs for coding genes in each sample. Genes with corrected p-value less than 0.05 and the absolute value of log2 (fold change) greater than or equal to 1 were assigned as significantly differentially expressed. Custom scripts on R software were used for clustering and heatmap analysis (https://www.r-project.org). In addition, the altered mRNA expression profile in shRelB vs. shCtrl was analyzed using KEGG pathway enrichment (https://david.ncifcrf.gov).

\section{Immunofluorescence}

PCa cells were seeded in confocal dishes at the density of $2 \times 10^{3}$ and were washed with cold $1 \times$ PBS and fixed within $4 \%$ paraformaldehyde in PBS for 15 minutes. Subsequently, the cells were permeabilized with $1 \times$ PBS containing $0.5 \%$ Triton X-100 for $20 \mathrm{~min}$ and then blocked for $30 \mathrm{~min}$. The cells were incubated with primary antibodies of RelB and PD-L1 at $4^{\circ} \mathrm{C}$ overnight then probed with Alexa Fluor ${ }^{\circledR} 647$ Conjugated (Cell Signaling Tech., USA) or Alexa Fluor ${ }^{\circledR} 488$ conjugated (abcam, UK) secondary antibody for $1 \mathrm{~h}$ at room temperature. After counterstaining with DAPI (Invitrogen) for $5 \mathrm{~min}$, fluorescence was visualized and captured using a TCS SP5 MP confocal microscope (Leica Microsystems, Inc., USA).

\section{NF-KB binding activity}

Nuclear proteins were exacted from PCa cells using a nuclear and cytoplasmic protein extraction kit (Beyotime Biotech.) according to the manufacture's instruction. The nuclear extracts $(50 \mu \mathrm{g})$ were subjected to a NF-KB binding activity kit containing the consensus sequence of the NF-KB element as a standard probe (abcom, USA) to measure the NF-KB binding activity based on the manufacture's protocol.

\section{Luciferase reporter assay}

A 2000-bp 5'-flanking region of the human CD274 gene containing a core promoter containing putative multiple NF-KB elements was cloned into pGL3 vector (Promega, USA) to drive the luciferase reporter gene. Subsequently, a functional NF-KB binding site was mutated using a site-directed mutagenesis system (Thermo Fisher, USA). The luciferase reporter constructs were co-transfected with $\beta$-gal into PC-3 cells using Lipofectamine (Invitrogen). After $48 \mathrm{~h}$, the luciferase activity was quantified by a Luciferase Assay System (Promega, USA) using a luminometer (Berthold Tech., Germany). The $\beta$-gal activity was 
quantified using microplate readers (BioTek, USA). The NF-kB-mediated transcriptional enhancement was estimated by $\beta$-gal-normalized luciferase response.

\section{ChIP}

RelB binding to the NF-kB elements in the human CD274 gene was examined by chromatin immunoprecipitation (ChIP) using a SimpleChIP Enzymatic Chromatin IP Kit (Cell Signaling Tech.). A RelB antibody (Santa Cruz Biotech., USA) was applied to pull down the chromatin from nuclear extracts isolated from PCa cells. Chromatin without antibody-pull down served as input control and IgG (Santa Cruz Biotech.) served as a negative antibody control. The pulled down fragments were quantified by PCR using relative primers (Table S1). Amounts of the pulled down fragments were assessed by normalizing with the input control.

\section{EMSA}

Electrophoretic mobility shift assay (EMSA) was further performed to confirm the specific RelB binding to the human CD274 promoter. A double-stranded DNA fragment containing the native or mutated NF-KB binding site was synthesized and annealed. The 3'- terminal of the upper strand was labeled using a probe biotin-labeling kit (Beyotime Biotech., China). EMSA was conducted by incubating the probes with PC-3 cells derived nuclear proteins within a chemiluminescent EMSA kit (Beyotime) according to the manufacturer's instruction. The unlabeled wild-type and mutated probes were used as competitors to quantify the specific binding activity. A RelB antibody was used to eliminate the binding activity. The EMSA image was visualized using a BioRad GelDoc XR + system. The sequences of the wild-type and mutant probes are listed in Table S1.

\section{RT-qPCR}

The mRNA levels RelB and PD-L1 were quantified by reverse transcription-quantitative PCR (RT-qPCR). Total RNA was extracted from PCa cells using the Tirzol reagent and CDNA was reversely transcribed relative mRNA using a PrimeScript ${ }^{\mathrm{TM}}$ RT reagent kit (Takara Inc., Japan) according to the manufacturer's instructions. cDNA was quantified by qPCR with SYBR Premix Ex Taq II (Takara Inc.) using a LightCycle System (Roche, USA). The mRNA level of the PD-L1 was estimated by normalizing with GAPDH mRNA. Sequences of the specific PCR primers for PD-L1 and GAPDH were listed in Table S1.

\section{Immunoblots}

PCa tumor tissues and cells were lysed in RIPA lysis buffer containing $1 \mathrm{mM}$ PMSF protein inhibitor (Santa Cruz Biotech.). Cellular and nuclear extracts $(50-100 \mu \mathrm{g})$ were separated on $10 \%$ SDSpolyacrylamide gels and then transferred to PVDF membranes. The membranes were subsequently incubated overnight at $4^{\circ} \mathrm{C}$ with the primary antibodies against RelA, RelB, PCNA, GAPDH (Cell Signaling Tech.), and anti-human PD-L1, anti-mouse PD-L1 (abcam). The membranes were washed three times with TBST buffer and incubated at room temperature for $2 \mathrm{~h}$ with HRP-conjugated secondary antibody (Santa Cruze Biotech. USA). The immunoblots were visualized using an enhanced chemiluminescence detection 
system (Bio-Rad, USA). The intensities of blots were quantified using Quantity One software and protein expression was normalized by loading controls such as $\beta$-actin and GAPDH.

\section{Bioinformatics}

Oncomine ${ }^{\mathrm{TM}}$ (https://www.oncomine.org) and TCGA (https://www.cancer.gov/about$\mathrm{nci} /$ organization/ccg/research/structural-genomics/tcga) datasets were examined to assess the association of RelA, RelB or PD-L1 expression profile with cancer progression and the correlation of RelA/RelB to PD-L1 was analyzed to estimate the possibility that NF-KB regulates PD-L1 expression in tumors.

\section{Statistics}

Results are presented as mean \pm standard deviation (SD) from at least three replicates. Significant differences between the experimental groups were analyzed by unpaired Student's t-test. One-way analysis of variance (ANOVA) followed by Dunnett's or Bonferroni's multiple comparison test using Prism (GraphPad, San Diego, USA). Statistical significance was accepted at $\mathrm{P}<0.05$.

\section{Results}

The constitutive levels PD-L1 and RelB are correlated with the aggressiveness of PCa. RelB has been shown that RelB associates with PCa and breast cancer progression [26, 27]. In consideration of the putative role of RelB in the regulation of cancer immune escape, we examined the Oncomine ${ }^{T M}$ and TCGA datasets for the enrichment of the potential relationship between PD-L1 and RelB signatures in cancer progression. PD-L1 uniquely expresses at high levels in multiple types of cancer tissues compared to their corresponding normal tissues (Fig. S1a). In addition, the correlation of PD-L1 with RelA and RelB in PCa was assessed. The results indicated that PD-L1 is highly associated with RelB compared to RelA in PCa tumor tissues, but no clear correlation was found in peritumor tissues (Fig. S1b). Interestingly, similar results were observed in breast cancer, indicating that the noncanonical NF-kB pathway is critical for the development of sex hormone relative cancers (Fig. S1C). In contrast, the correlation of PD-L1 with RelA is higher than RelB in lung cancer. There is no apparent relationship of PD-L1 with RelA or RelB in liver cancer (Fig. S1d-e).

To verify if the correlation of PD-L1 and RelB is also associated with the aggressiveness of PCa, tumor and normal prostate tissues were examined by IHC with specific antibodies against RelB and PD-L1. As expected, PD-L1 and RelB were consistently elevated, corresponding to increased patients' Gleason scores (Fig. 1a-d). The correlation between PD-L1 and RelB was associated with the pathological grads of tumor tissue samples (Fig. 1e). The results are consistent with the aspects from the Oncomine ${ }^{\text {TM }}$ database, suggesting that RelB may participate in the regulation of the $C D 274$ gene expression during PCa progression.

\section{RelB upregulates PD-L1 expression in PCa cells.}


Although the activation of RelA-based canonical NF-KB pathway is involved in chemo- or radio-therapy mediated PD-L1 induction in cancer cells [29,30], there is no evidence showing that the RelB-based noncanonical pathway contributes to PD-L1 expression in cancers. To elucidate the effect of RelB on PCa immune evasion, RelB was silenced in two aggressive AR-negative PCa cell lines (PC-3 and DU-145) using a lentiviral shRelB expressional construct. The reduction of NF-kB binding activity in RelB-silenced cells was confirmed. However, since other NF-KB members can also bind to the probe with the consensus NFKB sequence, the effect of RelB silence on total NF-KB binding activity was diluted, especially in DU-145 cells (Fig. 2a). RNA-Seq was applied to analyze mRNA expression profiles in the RelB-silenced PC-3 cells. The expression profiles of transcripts relevant to cytokines/chemokines production, inflammatory signaling pathway, immune response were selected as illustrated in Fig. 2b. Compared to the scramble control cells, most mRNA expression levels were reduced in response to the silence of RelB. KEGGenriched signaling pathway analysis showcased that the silence of RelB led to reductions in advanced PCa-associated cytokines/chemokines (Fig. S2a). Inflammation recognition-associated TLRs, NLRs and LTM were also declined in the RelB-silenced cells (Fig. S2b). Additionally, the silence of RelB in PCa cells led to inhibition of receptors associated with immune response (Fig. S2c). Furthermore, the mRNA expression profiles related to the immune response in the RelB-silenced cells were listed in Table S2. Remarkably, the mRNA level of the CD274 gene was declined in the RelB-silenced PC-3 cells.

To verify that RelB is able to regulate PD-L1 in PCa cells, the mRNA and protein levels of PD-L1 in the RelB-silenced PC-3 and DU-145 cells were quantified. RelB was efficiently knocked down in the two cell lines, but no change in RelA expression was observed. Consistent with the decline of RelB, the levels of PD-L1 mRNA and protein were also reduced (Fig. 2c-d). In addition, IFN- $\gamma$ remarkably induced PD-L1 expression in PC-3 cells, but the induction was alleviated when RelB was silenced (Fig. 2e). Consistent with the decreased level of nuclear RelB, the induction of PD-L1 protein was impeded (Fig. 2f). The Correlation of nuclear RelB and cytosolic PD-L1 was further confirmed by confocal microscope (Fig. 2g). These results suggested that IFN- $\gamma$ induces PD-L1 expression partially via RelB-mediated transcriptional activation.

\section{A proximal NF-KB element was identified to be responsive to RelB-mediated the CD274 gene transcriptional regulation.}

Although several lines of studies demonstrated that the NF-KB signaling regulates PD-L1 expression, there is a lack of experimental evidence that NF-KB directly regulates PD-L1 expression via the cis/trans transcriptional regulatory manner. To elucidate how RelB regulates the $C D 274$ gene expression, a 2000-bp 5 '-flanking fragment region containing a core promoter was cloned to drive the luciferase reporter gene expression. The luciferase activity was reduced in RelB-silenced PC-3 cells (Fig. 3a). Additionally, the IFN$\mathrm{Y}$-induced reporter response was declined when RelB was silenced (Fig. $3 \mathrm{~b}$ ). Three putative NF-KB binding sites (E1, E2, E3) located in the 5'-finking region were identified by analyzing the Jaspar transcription factor database (http://jaspar.genereg.net). Accordingly, a ChIP assay was performed to verify each

binding site using a RelB antibody. As shown in Fig. 3c, the proximal site (E3) appeared to be more susceptible to ChIP than E2 and distant E1 sites. Consistently, the amount of pull-down E3 element was 
reduced when chromatin extracted from RelB-silenced cells was used (Fig. 3d). Moreover, RelB binding to the E3 site was validated by EMSA. Nuclear extract was capable of shifting the probe containing the E3 site but not the mutant E3 site. An unlabeled probe was able to compete with the E3 binding, but an unlabeled mutant probe was found no such competition. In addition, the RelB antibody was able to reduce the $\mathrm{E} 3$ binding activity (Fig. $3 e$ ). Furthermore, Cell transfection with the mutated E3 site resulted in a reduction in the RelB-activated reporter response (Fig. S3a and Fig. 3f). Altogether, these results suggest that RelB transcriptionally regulates the human $C D 274$ gene expression via a proximal NF-kB element located in the core promoter region, which is conserved in human, mouse and rabbit (Fig. S4a-b).

\section{Tumor-derived RelB contributes to inactivation of $\mathrm{CD} 4{ }^{+}$and $\mathrm{CD} 8^{+} \mathrm{T}$ cells.}

Cancer immunotherapy aims to promote the cytotoxic effect of $\mathrm{T}$ lymphocytes within the tumor microenvironment. The signaling process is mainly relayed from $\mathrm{CD} 4^{+}$and $\mathrm{CD} 8^{+} \mathrm{T}$ cells by specific dendritic cells to optimize the immune response of T lymphocytes [31, 32]. Thus, it is important to define the immune responsiveness of T cells to RelB-depleted PC-3 cells. To this end, we collected blood samples and isolated the primary $T$ cells from healthy donors who participated in this study. The $T$ cells were activated by pretreating with anti-CD3, anti-CD28 and IL-2, and then co-cultured with RelB-silenced $\mathrm{PC}-3$ cells. The percentages of $\mathrm{CD} 4^{+}$and $\mathrm{CD} 8^{+} \mathrm{T}$ cells and their proliferation were quantified by flow cytometry with relative specific antibodies and CFSE dye. The results showed that the stimulation by CD3 and CD28 efficiently increased the numbers of T cells. Interestingly, co-culture with PC-3 cells significantly reduced the activated $\mathrm{CD} 4^{+}$and $\mathrm{CD} 8^{+} \mathrm{T}$ cells, but its effect was alleviated when RelB was depleted in PC3 cells (Fig. 4a-b). Consistently, the proliferation of $\mathrm{CD} 4^{+}$and $\mathrm{CD} 8^{+} \mathrm{T}$ cells was highly increased by the stimulation but further precluded after co-culturing with PC-3 cells. Nevertheless, the inhibitory effect of the PC-3 cells on the T-cell activation was favorably diminished by abrogating RelB (Fig. 4c-d).

Moreover, to testify whether tumorous RelB promotes T-cell immune compromise by increasing PD-L1, after co-culture, the survival of PC-3 cells was quantified by clonogenic assay. The activated T cells efficiently eliminated the PC-3 cell colony number compared to no co-culture control. Intriguingly, the T-cell immune response was further enhanced when RelB was silenced in PC-3 cells, suggesting that the high level of RelB in PC-3 cells contributes toward immune evasion (Fig. 4e).

\section{RelB deprivation enhances the immune checkpoint blockade by anti-PD-L1 inhibitor.}

Meanwhile, a CRISPR/Cas9 gene edition system was applied to knockout RelB in murine PCa RM-1 cells to verify that RelB contributes to immune evasion in vivo. Additionally, mouse PD-L1 was ectopically expressed in the RelB-KO cells to restore immune suppression (Fig. S3b and Fig. 5a). The NF-KB binding activity was measured in the gene manipulated RM-1 cells (Fig. 5b). The T cells were prepared from mouse spleens and then activated by CD3 and CD28 stimulation before co-cultured with RM- 1 cells (Fig. 5c). Consistent with the results from PC-3 cells above, the RelB-KO cells were more susceptible to T cells than control cells, but the T-cell activation was further eliminated by enforced expression of PD-L1 in RelB-KO cells (Fig. S5a). Similarly, co-culture with RelB-KO cells led to recovered CD $4^{+}$and $\mathrm{CD}^{+} \mathrm{T}$ cells 
compared to co-culture with RM-1 cells. In turn, the RelB-KO effect was further attenuated by expressing PD-L1 (Fig. S5b-c). In addition, the proliferation of CD $4^{+}$and $C D 8^{+} T$ cells further confirmed that the T-cell growth was virtually regulated by administrating RelB and PD-L1 in RM-1 cells (Fig. S5d-e).

Moreover, after co-culture with the activated T cells, RM-1 cells were treated with an anti-PD-L1 mAb. The results showed that the mAb strikingly enhanced the immune response of the T cells. Notably, RelB deprivation enhanced mAb therapeutic efficiency. Nevertheless, the enforced expression of PD-L1 in the RelB-KO cells led to partially rescues the cells (Fig. 5d). Correspondingly, the apoptotic cell rate was increased in RelB-KO cells treated with T-cell plus PD-L1 inhibitory mAb, but the effect was further alleviated as the PD-L1 level was elevated (Fig. 5e). These results suggest that the immune checkpoint blockade of PD-1/PD-L1 can be modulated by manipulating RelB.

\section{RelB contributes to PCa tumor immune evasion in mice.}

A mouse tumor xenograft model was applied to define the role of RelB in the immune checkpoint of PD1/PD-L1. RM-1, RM-1:RelB-KO and RM-1:RelB-KO/PD-L1 cell lines were used for tumor formation by subcutaneously injecting into mice. In the control group, 3-5 days after injection, tumors were formed and then rapidly grew to reach the maximal tumor volume $\left(3000 \mathrm{~mm}^{3}\right)$ within three weeks. In contrast, the tumor formation in the RelB-KO group was delayed and the tumor growth was also slow.

Nevertheless, the tumor growth in the RelB-KO/PD-L1 group was restored due to increased PD-L1 (Fig. 6ab). All mice were executed when the average tumor volume in the control group reached the maximum and tumor tissues were excised. Regardless of the endogenous mouse PD-L1, the levels of PD-L1 in RelBKO tumors were strikingly reduced, but the levels were raised in RelB-KO/PD-L1 tumors (Fig. 6c-d).

According to the manipulated PD-L1, the levels of CD4 and CD8 proteins increased via knock out of RelB, but the levels further decreased as PD-L1 was expressed (Fig. 6e). In addition, the mice serum samples were collected for T-cell activation (Fig. S6a). Compared to the control group, the numbers of $\mathrm{T}$ cells were increased in the RelB-KO group (Fig. S6b). After tumor formation, the amounts of CD $4^{+}$and $\mathrm{CD} 8^{+} \mathrm{T}$ cells slightly increased, but in turn, they were rapidly deceased as tumors consistently growing. Although CD $4^{+}$ and $\mathrm{CD}^{+} \mathrm{T}$ cells were high in the RelB-KO group, the cell numbers were declined as the increase of PD-L1 in tumor cells (Fig. 6f).

Moreover, to examine whether RelB deprivation also inhibits PCa metastasis by down-regulation of PD-L1, the three tumor cell lines were further injected into mice through tail veins. Three weeks after the injection, metastatic lung tumors were detected in the control group, but not in the groups injected with RelB-KO tumor cells irrespective of PD-L1 expressed in the cells, indicating that RelB is critical for PCa metastasis (Fig. 7a). Consistent with the reduction of RelB, the PD-L1 amounts also decreased in the RelB-KO group, but increased in the PD-L1 expressed group (Fig. 7b-C). Accordingly, CD4 ${ }^{+}$and $\mathrm{CD} 8^{+}$T-cell numbers in serums increased in mice injected with RelB-KO RM-1 cells, but the T-cell number dereased in injected mice by restoring PD-L1 in RM-1 cells (Fig. 7d). Taken together, the results from the present study delineated that the high levels of RelB in advanced PCa cells promote immune evasion by transcriptional upregulation of PD-L1, as illustrated in Fig. 7e. 


\section{Discussion}

Although the 5-year survival rates of PCa steadily increase in the United States, the mortality of PCa has been consistently increasing globally, particularly in East Asia, like China [2]. AR-negative metastatic PCa displays resistance to most common treatments and leads to poor prognosis, particularly devastating bone metastasis appears to be a salient daunting challenge in the control of $\mathrm{PCa}$ [33]. Thus, the comprehensive therapeutic strategy in combination with innovative technologies urgently needs to conquer intractable malignant PCa. In this regard, the advantages of novel cancer immunotherapy have emerged as a prospective approach to treat worsened tumors, including advanced PCa [7, 34].

Mounting evidence demonstrated that immune checkpoint inhibitors have significantly improved overall survival for subsets of patients with malignant tumors that mostly resist the traditional anticancer therapies $[35,36]$. For instance, the therapeutic effects of the blockade of PD-1/PDL-1 and CTLA-4 checkpoints have been adopted to treat various cancers [37, 38]. PD-L1 uniquely expresses at the high levels in cancers, thereby therapies targeting PD-1/PD-L1 have been shown to promote remarkable antitumor immunity and acquire promising therapeutic outcomes for several malignant tumors $[11,39]$. Likewise, PD-L1 profoundly expresses in tumor tissues from castration-resistant prostate cancer (CRPC) patients, suggesting that PD-L1 is associated with PCa progression [40]. Although anti-PD-1 treatment has shown therapeutic results for treating primary PCa, the combination of anti-PD-1 mAb with myeloidderived suppressor cell (MDSC)-targeted therapy has received a robust synergistic therapeutic response in the treatment of CRPC [41]. Nevertheless, lack of response to anti-PD-L1 agents in the treatment of metastatic urothelial cancer and CRPC indicated stromal TGF- $\beta$ dampening the PD-1/PD-L1 blockade therapy, indicating that the existence of alternative immunosuppressive mechanisms provided from the tumor microenvironment $[42,43]$.

It is recognized that pro-inflammatory cytokines produced in the tumor microenvironment induce PD-L1 expression in tumor cells $[44,45]$. In particular, IFN- $\gamma$ has been defined as a favorable adaptive immune resistant inducer. Other oncogenic cytokines were also involved in inducing PD-L1 expression, including TGF- $\beta$, IL-6, IL-10, and IL-17 [15]. Mechanistically, the expression of PD-L1 in tumor cells can be regulated through transcriptional and posttranscriptional regulations [14]. Several cytokine/chemokine-inducible transcription factors have been shown to participate in the regulation of PD-L1, such as Myc, NF-KB, Stat3, and Jun/Ap-1 $[15,46]$. Additionally, tumor-suppressive miR-34a appeared to directly downregulate PD-L1 [47], while oncogenic miR-21 seemed to indirectly upregulate PD-L1 via activation of PI3K-Akt signal axis by inhibiting PTEN [48]. Furthermore, a recent study demonstrated that AKT upregulates PDL1 by phosphorylating $\beta$-catenin and promotes glioblastoma immune evasion [49].

Transcriptional regulation plays a pivotal role in PCa progression. AR-mediated transcriptional regulation plays a fundamental role in the promotion of AR-dependent PCa [50]. Nevertheless, PCa patients frequently received androgen-deprivation and AR-inhibitory therapies, and the AR response is ultimately eradicated in the developed AR-negitive malignancy [51, 52]. Notably, NF-KB-mediated transcriptional regulation, in turn, to be activated in CRPC, functionally sustains PCa progression at the androgen-free 
condition [53]. Although the activation of the RelA-based canonical NF-KB pathway has been involved in $\mathrm{PCa}$ progression and therapeutic resistance $[54,55]$, the role of the RelB-based noncanonical NF-KB pathway is underestimated.

RelA has been reported to upregulate PD-L1 in tumor cells in response to TNF-a and LPS stimulations or by cooperation with RB phosphorylation $[17,56,57]$. Nevertheless, there is a lack of current evidence that p50/RelA directly binds to the NF-KB element located in the CD274 promoter. Recently, Antonangeli et al. have predicted the canonical NF-KB consensus sequence in the $C D 274$ promoter region as 5'GGGRNWYYCC-3' (where R: A/G, W: A/T, Y: C/T, N: any base); however, the proposed site has been not fully validated yet [58]. The present study used the standard NF-KB consensus sequence (5'GGGRNYYYCC-3') for searching the potential NF-KB binding site in the CD274 promoter. Three putative sites were validated using a systemic cis/trans transcriptional regulatory approach. The results delineated that a proximal NF-KB enhancer element located in the core promoter region is responsive to RelB-mediated PCa immune evasion by upregulating PD-L1. Consistently, the silence of tumorous RelB led to enhanced T-cell immunity in the suppression of PCa.

Notably, the RelB-based noncanonical NF-kB pathway has been implicated in diverse biological processes, including immunogenicity and tumorigenicity [59]. RelB function is critical for normal B-cell maturation and lymphoid organogenesis [60, 61]. BAFF-NIK-p52/RelB axis is essential for B-cell survival by upregulating Bcl-2 and Bcl-xl [62]. Additionally, TRAF3- or NIK-deficiency appeared to preclude T-cell function by inhibiting the noncanonical NF-KB pathway $[63,64]$. Intriguingly, the results from this study,

for the first time, elucidated that tumor-derived RelB hampers T-cell function by upregulating PD-L1. Thus, the implication of RelB in both immune cells and tumor cells has prospected to be a major concern for tumor immunotherapy. Expectedly, the insight into RelB-mediated PD-L1 overexpression is anticipated to provide a promising approach for enhancing immune checkpoint blockade therapy through the administration of RelB.

\section{Conclusion}

In summary, this study delineates that RelB participates in the regulation of PD-L1 in PCa cells. RelB and PD-L1 highly express in advanced PCa and contribute to immune evasion. The silence of RelB led to reduced PD-L1 expression and enhanced T-cell immune response. RelB upregulates PD-L1 in response to cytokines, mainly through binding to a proximal NF-kB element located in the core promoter region of the $C D 274$ gene.

\section{Abbreviations}

PCa, prostate cancer; CRPC, castration-resistant prostate cancer; TCGA, the cancer genome atlas; PD-1, programmed death receptor, PD-L1, ligand of PD-1; CTLA-4, cytotoxic T lymphocyte-associatedantigen4; NF-kB, nuclear factor kappa light chain enhancer of activated B cells; IFN-y, human interferongamma; TNF-a, tumour necrosis factoralpha; IL-2, interleukin-2;shRNA, small hairpin RNA; KO, 
knockout; IHC, immunohistochemistry; RNAseq, RNA sequencing; RT-qPCR, reverse transcriptionquantitative PCR; ChIP, chromatin immuneprecipitation; EMSA, electrophoretic mobility shift assay; RBC, red blood cells.

\section{Declarations}

\section{Acknowledgments}

The authors thank Dr. Hongbing Shen, Director of Cancer Research Cancer at Nanjing Medical University, to provide most research resources supporting this study.

\section{Funding}

This study was supported by the National Natural Science Foundation of China Research Grants (Nos. 81572742 and 81972742) and the National Program on Key Research Project of China (No. 2016YFC0905900) to Xu Y; National Nature Science Foundation of China Young Investigator Award (No. 81602681) and China Postdoctoral Science Foundation (No. 2017M621681) to Zhang Y; Jiangsu Provincial Key Research Development Program (BE 2017759) to Qin Zhang.

\section{Author information}

\section{Affiliations}

The Affiliated Cancer Hospital of Nanjing Medical University \& Jiangsu Cancer

Hospital \& Jiangsu Institute of Cancer Research, Nanjing 210009, China

Yanyan Zhang, Yuanyuan Du, Fan Xu, Wenbo Sun, Zhi Xu, Xiumei Wang, Peipei Qian, Qin, Zhang, Jifeng Fengand Yong $\mathrm{Xu}$

Jiangsu Key Lab of Cancer Biomarkers, Prevention and Treatment, Nanjing Medical University, Nanjing 211166, China

Shuyi Zhu and Yong Xu

\section{Authors' contributions}

Conception and design: $Y Z$ and $Y X$; Development of methodology: $Y Z$ and SZ; Acquisition of data: $Y Z, S Z$ and YD; Analysis and interpretation of data: YZ, SZ and YX; Administrative, technical, or material support: FX, ZY, WS, XW and PQ; Writing, review, and/or revision of the manuscript: YZ and YX; Study supervision: $Y X, Q Z$ and JF.

\section{Corresponding authors}

Qin Zhang, Jifeng Feng and Yong Xu 


\section{Patients consent for this study}

Written informed consent was obtained from patients enrolled in this study based on the guidelines of human study declaration issued by the medical ethics committee of Nanjing Medical University.

\section{Ethics approval}

Human participants involved in this study were approved by The Ethics Committee of Nanjing Medical University, (2018) No. 565. All animal experiments were performed according to the Institutional Animal Care and Use approved by the Research Committee of Nanjing Medical University (No. IACUC-1901031).

\section{Availability of data and materials}

All data relevant to this study are included in the article and accessible onlinesupplemental information. Data are available in a public, open access repository. Materials included in this study will be available for reasonable requests to the corresponding author, Prof. Yong Xu.

\section{Competing interests}

The authors declare no competing interests.

\section{References}

1. Culp MB, Soerjomataram I, Efstathiou JA, Bray F, Jemal A. Recent Global Patterns in Prostate Cancer Incidence and Mortality Rates. Eur Urol. 2020;77:38-52.

2. Miller KD, Nogueira L, Mariotto AB, Rowland JH, Yabroff KR, Alfano CM, Jemal A, Kramer JL, Siegel RL. Cancer treatment and survivorship statistics, 2019. CA Cancer J Clin. 2019;69:363-85.

3. Yang Y. Cancer immunotherapy: harnessing the immune system to battle cancer. J Clin Invest. 2015;125:3335-7.

4. Kwek SS, Cha E, Fong L. Unmasking the immune recognition of prostate cancer with CTLA4 blockade. Nat Rev Cancer. 2012;12:289-97.

5. Pitt JM, Vetizou M, Daillere R, Roberti MP, Yamazaki T, Routy B, Lepage P, Boneca IG, Chamaillard M, Kroemer G, Zitvogel L. Resistance Mechanisms to Immune-Checkpoint Blockade in Cancer: TumorIntrinsic and -Extrinsic Factors. Immunity. 2016;44:1255-69.

6. Goodman A, Patel SP, Kurzrock R. PD-1-PD-L1 immune-checkpoint blockade in B-cell lymphomas. Nat Rev Clin Oncol. 2017;14:203-20.

7. Brahmer JR, Tykodi SS, Chow LQ, Hwu WJ, Topalian SL, Hwu P, Drake CG, Camacho LH, Kauh J, Odunsi K, et al. Safety and activity of anti-PD-L1 antibody in patients with advanced cancer. N Engl J Med. 2012;366:2455-65.

8. Okazaki T, Chikuma S, Iwai Y, Fagarasan S, Honjo T. A rheostat for immune responses: the unique properties of PD-1 and their advantages for clinical application. Nat Immunol. 2013;14:1212-8. 
9. Zak KM, Grudnik P, Magiera K, Domling A, Dubin G, Holak TA. Structural Biology of the Immune Checkpoint Receptor PD-1 and Its Ligands PD-L1/PD-L2. Structure. 2017;25:1163-74.

10. Webb JR, Milne K, Kroeger DR, Nelson BH. PD-L1 expression is associated with tumor-infiltrating T cells and favorable prognosis in high-grade serous ovarian cancer. Gynecol Oncol. 2016;141:293302.

11. Casey SC, Tong L, Li Y, Do R, Walz S, Fitzgerald KN, Gouw AM, Baylot V, Gutgemann I, Eilers M, Felsher DW. MYC regulates the antitumor immune response through CD47 and PD-L1. Science. 2016;352:227-31.

12. Tumeh PC, Harview CL, Yearley JH, Shintaku IP, Taylor EJ, Robert L, Chmielowski B, Spasic M, Henry G, Ciobanu V, et al. PD-1 blockade induces responses by inhibiting adaptive immune resistance. Nature. 2014;515:568-71.

13. Platanias LC. Mechanisms of type-I- and type-II-interferon-mediated signalling. Nat Rev Immunol. 2005;5:375-86.

14. Sun C, Mezzadra R, Schumacher TN. Regulation and Function of the PD-L1 Checkpoint. Immunity. 2018;48:434-52.

15. Cha JH, Chan LC, Li CW, Hsu JL, Hung MC. Mechanisms Controlling PD-L1 Expression in Cancer. Mol Cell. 2019;76:359-70.

16. Ben-Neriah Y, Karin M. Inflammation meets cancer, with NF-kappaB as the matchmaker. Nat Immunol. 2011;12:715-23.

17. Asgarova A, Asgarov K, Godet Y, Peixoto P, Nadaradjane A, Boyer-Guittaut M, Galaine J, Guenat D, Mougey $\mathrm{V}$, Perrard J, et al. PD-L1 expression is regulated by both DNA methylation and NF-kB during EMT signaling in non-small cell lung carcinoma. Oncoimmunology. 2018;7:e1423170.

18. Karin M. NF-kappaB and cancer: mechanisms and targets. Mol Carcinog. 2006;45:355-61.

19. Hayden MS, Ghosh S. Shared principles in NF-kappaB signaling. Cell. 2008;132:344-62.

20. Sun SC. Non-canonical NF-kappaB signaling pathway. Cell Res. 2011;21:71-85.

21. van Delft MA, Huitema LF, Tas SW. The contribution of NF-kappaB signalling to immune regulation and tolerance. Eur J Clin Invest. 2015;45:529-39.

22. Burkly L, Hession C, Ogata L, Reilly C, Marconi LA, Olson D, Tizard R, Cate R, Lo D. Expression of relB is required for the development of thymic medulla and dendritic cells. Nature. 1995;373:531-6.

23. Weih F, Carrasco D, Durham SK, Barton DS, Rizzo CA, Ryseck RP, Lira SA, Bravo R. Multiorgan inflammation and hematopoietic abnormalities in mice with a targeted disruption of RelB, a member of the NF-kappa B/Rel family. Cell. 1995;80:331-40.

24. Sun SC. The non-canonical NF-kappaB pathway in immunity and inflammation. Nat Rev Immunol. 2017; 17:545-58.

25. Ge QL, Liu SH, Ai ZH, Tao MF, Ma L, Wen SY, Dai M, Liu F, Liu HS, Jiang RZ, et al. RelB/NF-kappaB links cell cycle transition and apoptosis to endometrioid adenocarcinoma tumorigenesis. Cell Death Dis. 2016;7:e2402. 
26. Wang M, Zhang Y, Xu Z, Qian P, Sun W, Wang X, Jian Z, Xia T, Xu Y, Tang J. RelB sustains endocrine resistant malignancy: an insight of noncanonical NF-kappaB pathway into breast Cancer progression. Cell Commun Signal. 2020;18:128.

27. Xu Y, Josson S, Fang F, Oberley TD, St Clair DK, Wan XS, Sun Y, Bakthavatchalu V, Muthuswamy A. St Clair WH: RelB enhances prostate cancer growth: implications for the role of the nuclear factorkappaB alternative pathway in tumorigenicity. Cancer Res. 2009;69:3267-71.

28. Zhang Y, Xu Z, Ding J, Tan C, Hu W, Li Y, Huang W, Xu Y. HZO8 suppresses RelB-activated MnSOD expression and enhances Radiosensitivity of prostate Cancer cells. J Exp Clin Cancer Res. 2018;37:174.

29. Peng J, Hamanishi J, Matsumura N, Abiko K, Murat K, Baba T, Yamaguchi K, Horikawa N, Hosoe Y, Murphy SK, et al. Chemotherapy Induces Programmed Cell Death-Ligand 1 Overexpression via the Nuclear Factor-kappaB to Foster an Immunosuppressive Tumor Microenvironment in Ovarian Cancer. Cancer Res. 2015;75:5034-45.

30. Wang W, Chapman NM, Zhang B, Li M, Fan M, Laribee RN, Zaidi MR, Pfeffer LM, Chi H, Wu ZH. Upregulation of PD-L1 via HMGB1-Activated IRF3 and NF-kappaB Contributes to UV RadiationInduced Immune Suppression. Cancer Res. 2019;79:2909-22.

31. Borst J, Ahrends T, Babala N, Melief CJM, Kastenmuller W. CD4(+) T cell help in cancer immunology and immunotherapy. Nat Rev Immunol. 2018;18:635-47.

32. Farhood B, Najafi M, Mortezaee K. CD8(+) cytotoxic T lymphocytes in cancer immunotherapy: A review. J Cell Physiol. 2019;234:8509-21.

33. Berish RB, Ali AN, Telmer PG, Ronald JA, Leong HS. Translational models of prostate cancer bone metastasis. Nat Rev Urol. 2018;15:403-21.

34. Isaacsson Velho P, Antonarakis ES. PD-1/PD-L1 pathway inhibitors in advanced prostate cancer. Expert Rev Clin Pharmacol. 2018;11:475-86.

35. Qin S, Xu L, Yi M, Yu S, Wu K, Luo S. Novel immune checkpoint targets: moving beyond PD-1 and CTLA-4. Mol Cancer. 2019;18:155.

36. Palucka AK, Coussens LM. The Basis of Oncoimmunology. Cell. 2016;164:1233-47.

37. Darvin P, Toor SM, Sasidharan Nair V, Elkord E. Immune checkpoint inhibitors: recent progress and potential biomarkers. Exp Mol Med. 2018;50:1-11.

38. Ott PA, Hodi FS, Robert C. CTLA-4 and PD-1/PD-L1 blockade: new immunotherapeutic modalities with durable clinical benefit in melanoma patients. Clin Cancer Res. 2013;19:5300-9.

39. Chen L, Han X. Anti-PD-1/PD-L1 therapy of human cancer: past, present, and future. J Clin Invest. 2015;125:3384-91.

40. Gevensleben H, Dietrich D, Golletz C, Steiner S, Jung M, Thiesler T, Majores M, Stein J, Uhl B, Muller S, et al. The Immune Checkpoint Regulator PD-L1 Is Highly Expressed in Aggressive Primary Prostate Cancer. Clin Cancer Res. 2016;22:1969-77. 
41. Lu X, Horner JW, Paul E, Shang X, Troncoso P, Deng P, Jiang S, Chang Q, Spring DJ, Sharma P, et al. Effective combinatorial immunotherapy for castration-resistant prostate cancer. Nature. 2017;543:728-32.

42. Mariathasan S, Turley SJ, Nickles D, Castiglioni A, Yuen K, Wang Y, Kadel EE III, Koeppen H, Astarita JL, Cubas R, et al. TGFbeta attenuates tumour response to PD-L1 blockade by contributing to exclusion of T cells. Nature. 2018;554:544-8.

43. Jiao S, Subudhi SK, Aparicio A, Ge Z, Guan B, Miura Y, Sharma P. Differences in Tumor Microenvironment Dictate T Helper Lineage Polarization and Response to Immune Checkpoint Therapy. Cell. 2019;179:1177-90 e1113.

44. Lim SO, Li CW, Xia W, Cha JH, Chan LC, Wu Y, Chang SS, Lin WC, Hsu JM, Hsu YH, et al. Deubiquitination and Stabilization of PD-L1 by CSN5. Cancer Cell. 2016;30:925-39.

45. Chen S, Crabill GA, Pritchard TS, McMiller TL, Wei P, Pardoll DM, Pan F, Topalian SL. Mechanisms regulating PD-L1 expression on tumor and immune cells. J Immunother Cancer. 2019;7:305.

46. Ritprajak P, Azuma M. Intrinsic and extrinsic control of expression of the immunoregulatory molecule PD-L1 in epithelial cells and squamous cell carcinoma. Oral Oncol. 2015;51:221-8.

47. Anastasiadou E, Stroopinsky D, Alimperti S, Jiao AL, Pyzer AR, Cippitelli C, Pepe G, Severa M, Rosenblatt J, Etna MP, et al. Epstein-Barr virus-encoded EBNA2 alters immune checkpoint PD-L1 expression by downregulating miR-34a in B-cell lymphomas. Leukemia. 2019;33:132-47.

48. Xi J, Huang Q, Wang L, Ma X, Deng Q, Kumar M, Zhou Z, Li L, Zeng Z, Young KH, et al. miR-21 depletion in macrophages promotes tumoricidal polarization and enhances PD-1 immunotherapy. Oncogene. 2018;37:3151-65.

49. Du L, Lee JH, Jiang H, Wang C, Wang S, Zheng Z, Shao F, Xu D, Xia Y, Li J, et al: beta-Catenin induces transcriptional expression of PD-L1 to promote glioblastoma immune evasion. J Exp Med 2020, 217.

50. Dai C, Heemers H, Sharifı N. Androgen Signaling in Prostate Cancer. Cold Spring Harb Perspect Med 2017, 7.

51. Laudato S, Aparicio A, Giancotti FG. Clonal Evolution and Epithelial Plasticity in the Emergence of AR-Independent Prostate Carcinoma. Trends Cancer. 2019;5:440-55.

52. Sowalsky AG, Ye H, Bhasin M, Van Allen EM, Loda M, Lis RT, Montaser-Kouhsari L, Calagua C, Ma F, Russo JW, et al. Neoadjuvant-Intensive Androgen Deprivation Therapy Selects for Prostate Tumor Foci with Diverse Subclonal Oncogenic Alterations. Cancer Res. 2018;78:4716-30.

53. Jin R, Yi Y, Yull FE, Blackwell TS, Clark PE, Koyama T, Smith JA Jr, Matusik RJ. NF-kappaB gene signature predicts prostate cancer progression. Cancer Res. 2014;74:2763-72.

54. Grosset AA, Ouellet V, Caron C, Fragoso G, Barres V, Delvoye N, Latour M, Aprikian A, Bergeron A, Chevalier $S$, et al. Validation of the prognostic value of NF-kappaB p65 in prostate cancer: $A$ retrospective study using a large multi-institutional cohort of the Canadian Prostate Cancer Biomarker Network. PLoS Med. 2019;16:e1002847.

55. Jeong JH, Park SJ, Dickinson SI, Luo JL. A Constitutive Intrinsic Inflammatory Signaling Circuit Composed of miR-196b, Meis2, PPP3CC, and p65 Drives Prostate Cancer Castration Resistance. Mol 
Cell. 2017;65:154-67.

56. Jin X, Ding D, Yan Y, Li H, Wang B, Ma L, Ye Z, Ma T, Wu Q, Rodrigues DN, et al. Phosphorylated RB Promotes Cancer Immunity by Inhibiting NF-kappaB Activation and PD-L1 Expression. Mol Cell. 2019;73:22-35 e26.

57. Li H, Xia JQ, Zhu FS, Xi ZH, Pan CY, Gu LM, Tian YZ. LPS promotes the expression of PD-L1 in gastric cancer cells through NF-kappaB activation. J Cell Biochem. 2018;119:9997-10004.

58. Antonangeli F, Natalini A, Garassino MC, Sica A, Santoni A, Di Rosa F. Regulation of PD-L1 Expression by NF-kB in Cancer. Frontiers in Immunology 2020, 11.

59. Cildir G, Low KC, Tergaonkar V. Noncanonical NF-kappaB Signaling in Health and Disease. Trends Mol Med. 2016;22:414-29.

60. Liu Z, Mar KB, Hanners NW, Perelman SS, Kanchwala M, Xing C, Schoggins JW, Alto NM. A NIK-SIX signalling axis controls inflammation by targeted silencing of non-canonical NF-kappaB. Nature. 2019;568:249-53.

61. Mukherjee T, Chatterjee B, Dhar A, Bais SS, Chawla M, Roy P, George A, Bal V, Rath S, Basak S. A TNFp100 pathway subverts noncanonical NF-kappaB signaling in inflamed secondary lymphoid organs. EMBO J. 2017;36:3501-16.

62. Claudio E, Brown K, Park S, Wang H, Siebenlist U. BAFF-induced NEMO-independent processing of NF-kappa B2 in maturing B cells. Nat Immunol. 2002;3:958-65.

63. Chang JH, Hu H, Jin J, Puebla-Osorio N, Xiao Y, Gilbert BE, Brink R, Ullrich SE, Sun SC. TRAF3 regulates the effector function of regulatory $\mathrm{T}$ cells and humoral immune responses. J Exp Med. 2014;211:137-51.

64. Mair F, Joller S, Hoeppli R, Onder L, Hahn M, Ludewig B, Waisman A, Becher B. The NFkappaBinducing kinase is essential for the developmental programming of skin-resident and IL-17-producing gammadelta T cells. Elife 2015, 4.

\section{Figures}


Figure 1

A

A Normal
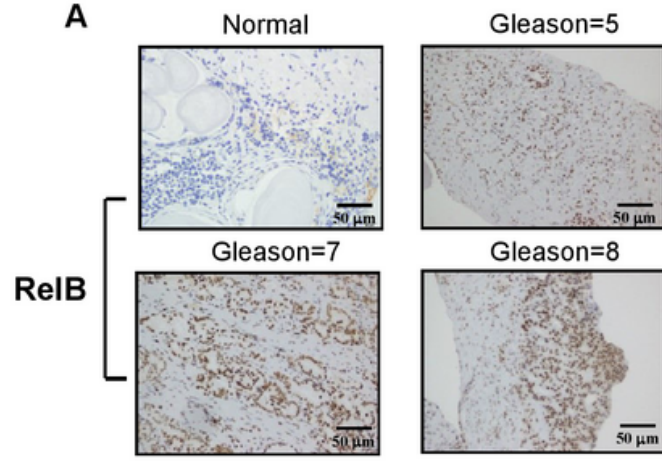

C

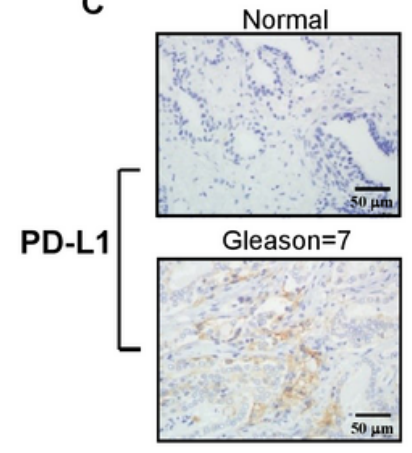

Gleason $=5$

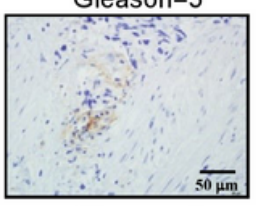

Gleason $=8$

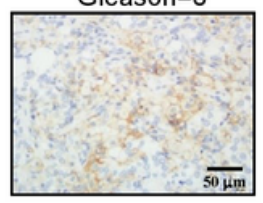

Gleason $=6$

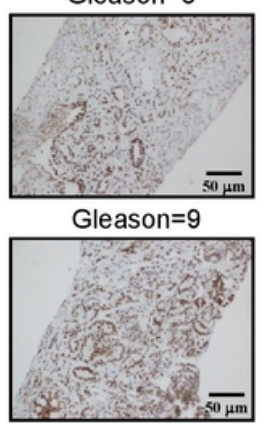

Gleason $=6$

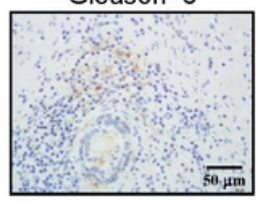

Gleason $=9$

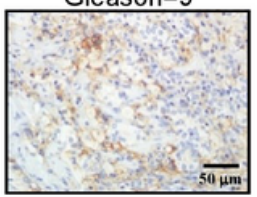

B

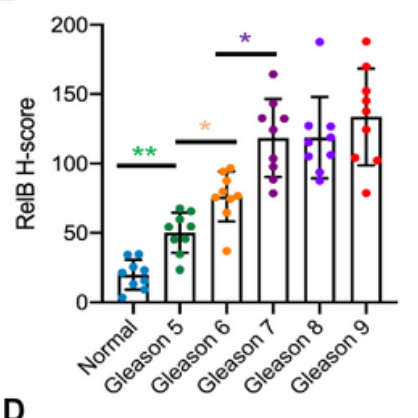

D

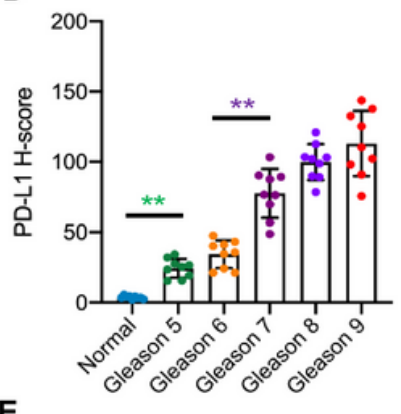

E

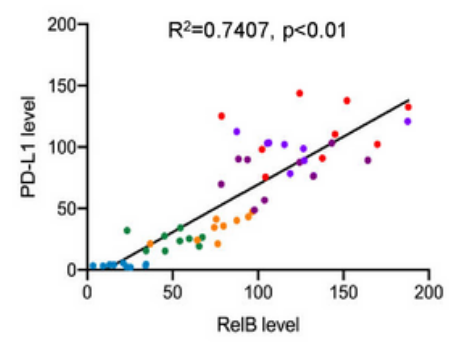

Figure 1

PD-L1 is correlated with RelB in PCa progression. a-b Normal prostate and PCa tissues were examined to determine the association between RelB expression and patients' Gleason scores by IHC. c-d Correspondingly, the association between PD-L1 expression and patients' Gleason scores was analyzed. e The linear regression analysis determines the correlation of RelB and PD-L1 in PCa progression. The Hscores of IHC were plotted and $*(p<0.05)$ and $* *(p<0.01)$ present the significances between two groups as indicated. 


\section{Figure 2}
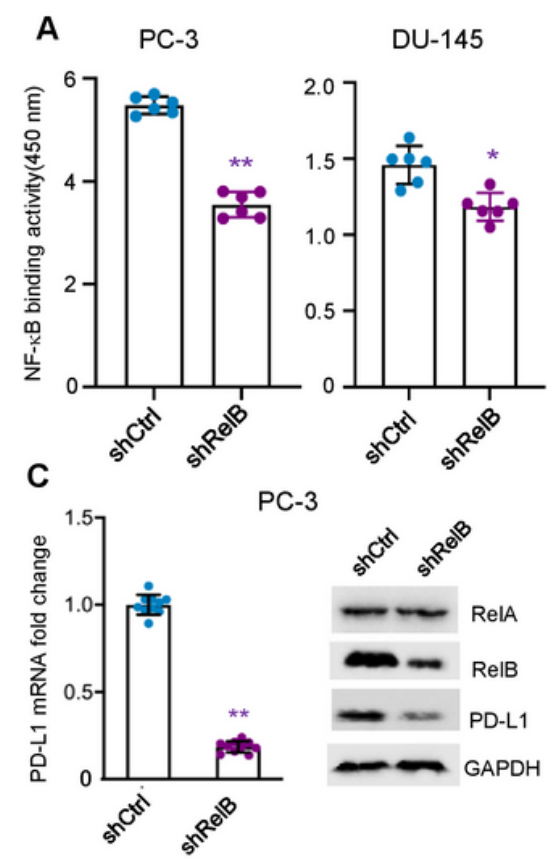

PC-3
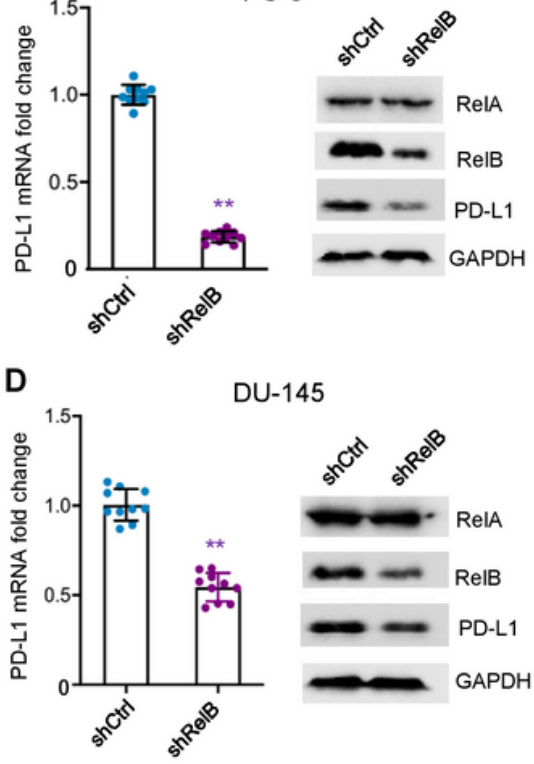

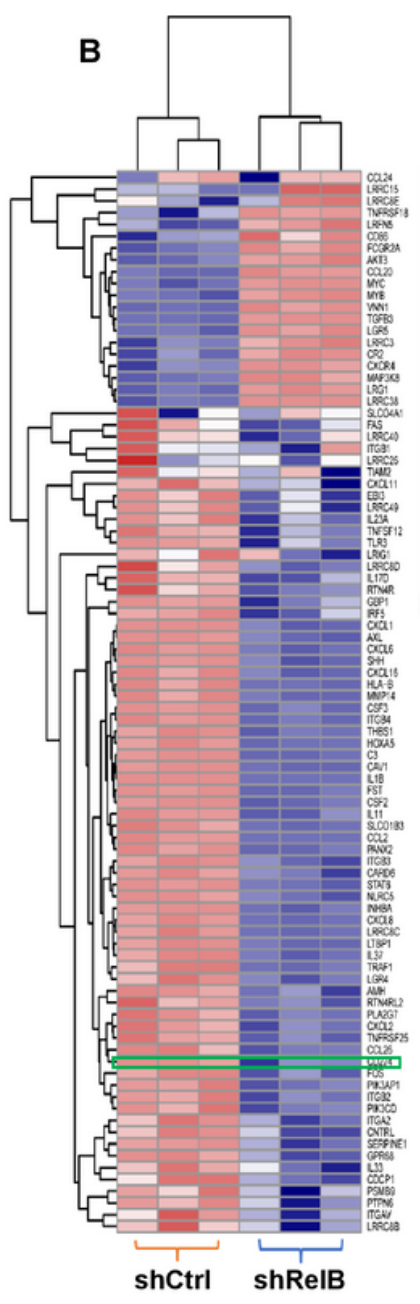

E

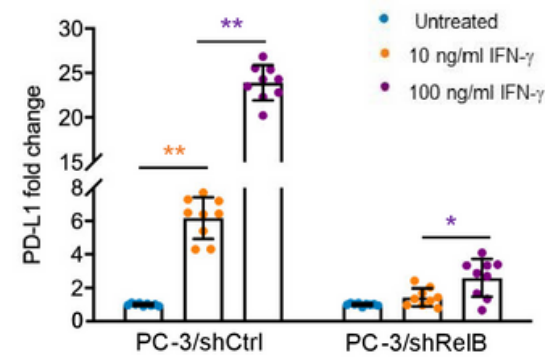

$\mathbf{F}$

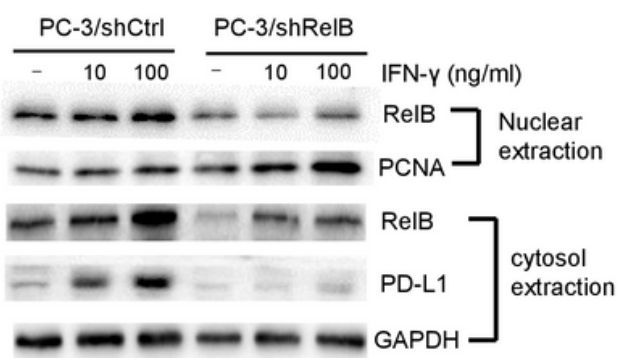

G

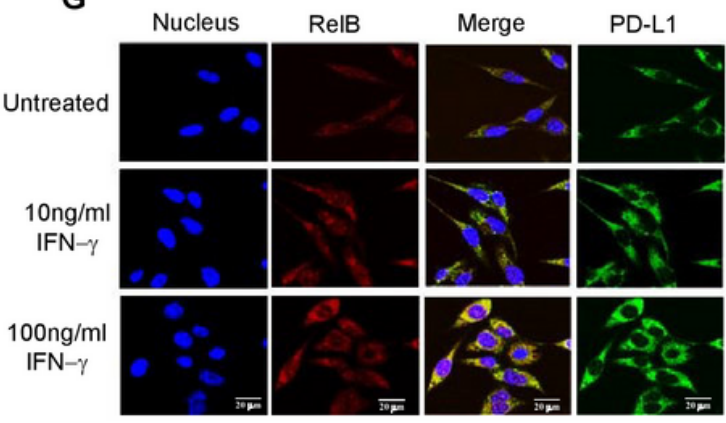

\section{Figure 2}

RelB regulates PD-L1 in PCa cells. a RelB was silenced in PC-3 and DU-145 cells using a lentiviral shRNA specifically targeting endogenic RelB. The NF-kB binding activities in RelB-silenced cells (shRelB) vs. scramble sequence (shCtrl) were measured using an ELISA kit with a standard probe containing the consensus NF-KB element. b mRNA expression profiles of RelB-silenced cells vs. control cells were examined by RNA sequencing. The relative levels of inflammatory and immune responsive transcripts were analyzed, as indicated by the heatmap. The signature of CD274 gene expression was indicated in a green box. c-d The levels of RelA, RelB and PD-L1 mRNA and proteins were quantified using RT-qPCR and immunoblots by normalizing with GAPDH. e PC-3 cells were treated with different doses of IFN-y as indicated. The stimulated PD-L1 mRNA expression was quantified by RT-qPCR with a GAPDH normalization. f After IFN- $y$ treatment, the levels of nuclear RelB and relative cytosolic PD-L1 in PC-3 cells were measured by immunoblots. PCNA serves as an internal control of nuclear fraction and GAPDH as a loading control of cytosolic fraction. g Accordingly, RelB nuclear translocation and relative PD-L1 in the cytosol were confirmed using a confocal microscope. $*(p<0.05)$ and ${ }^{*}(p<0.01)$ show the significances between the two groups as indicated. 
Figure 3
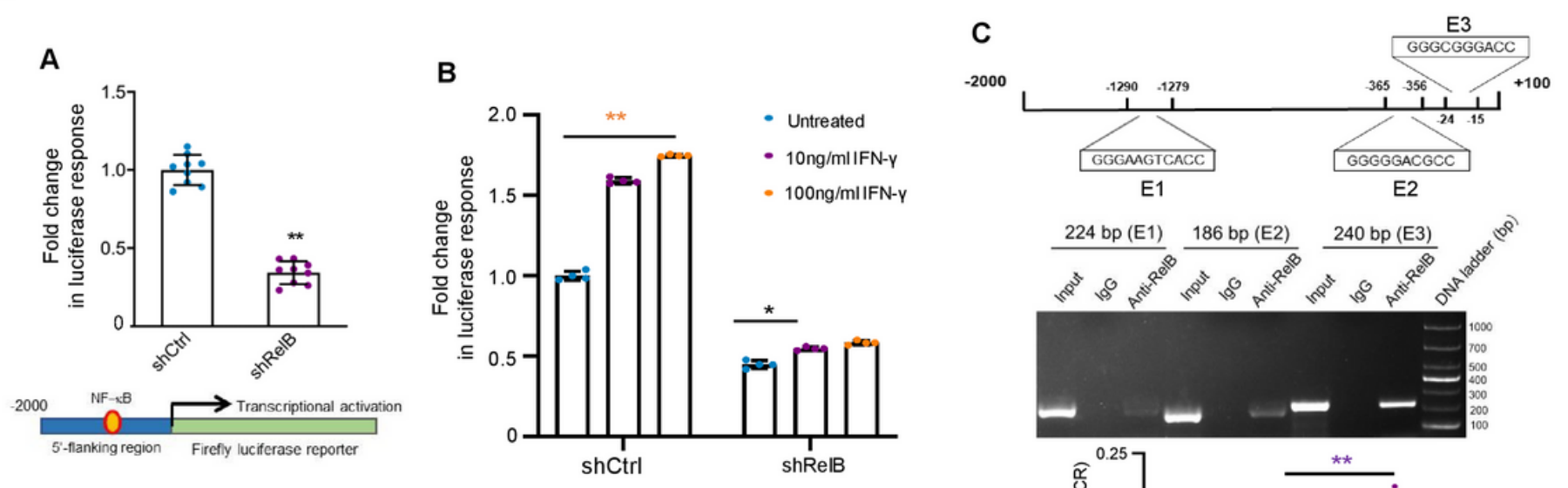

E1 E2

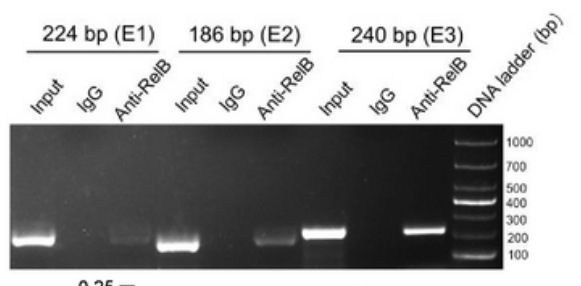

5 -flanking region Firefly luciferase reporter

D
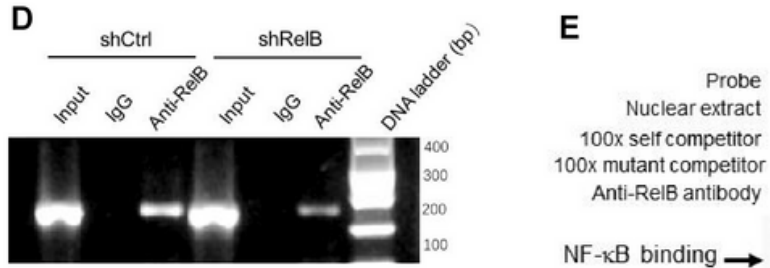

NF- $\mathrm{kB}$ binding $\rightarrow$
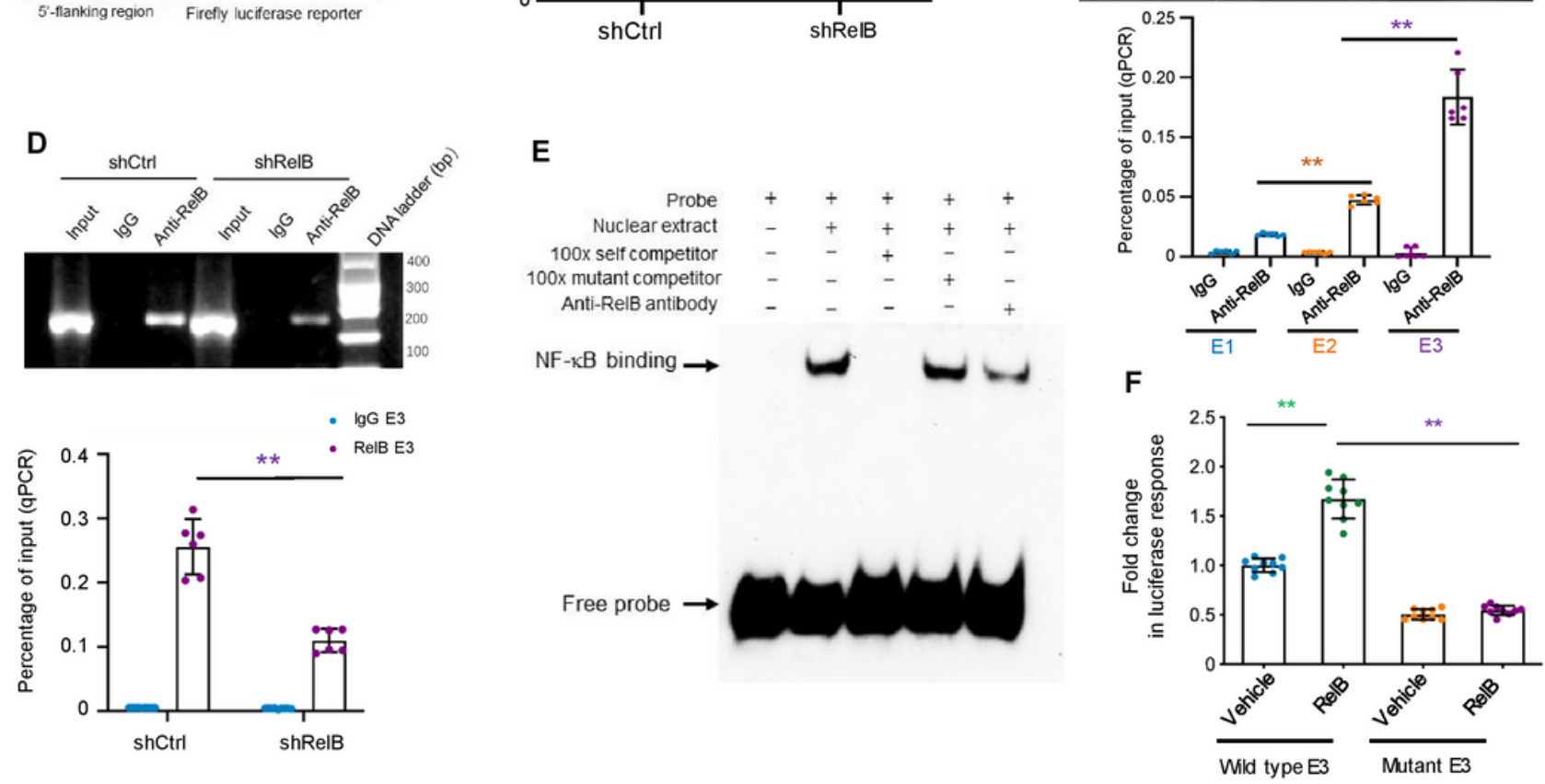

\section{Figure 3}

RelB regulates PD-L1 in PC-3 cells. a A 2000-bp flanking region of the human CD274 gene containing putative NF-KB elements and a core promoter was cloned to drive the luciferase reporter gene, as indicated in the lower panel. The reporter construct was transfected into PC-3 cells with different levels of RelB and the transcriptional activity was estimated by $\beta$-gal-normalized reporter responses. $b$ After cell transfection, the cells were treated with IFN- $y$ as indicated. The IFN- $\gamma$-mediated transcriptional induction was analyzed by the reporter assay. c Chromatin was precipitated using a RelB antibody, DNA fragments containing three putative RelB elements (E1, E2, E3) were quantified by regular PCR (upper panel) and quantitative PCR (lower panel). Chromatins without the IP procedure were amplified as input controls. IgG was used as a negative antibody control. $d$ Chromatins derived from RelB-silenced were pulled down and the reduction in E3 fragment was quantified. e A 24-bp double-stranded DNA fragment containing the E3 element was synthesized for the preparation of a EMSA probe with biotin-labeling. Nuclear extract was incubated with the probe and the specific NF-kB binding was determined by EMSA with a self competitor. Additionally, the NF-KB element in the E3 fragment was mutated as a mutant competitor (shown in Fig. S3a). Furthermore, the RelB antibody was used to reduce the E3 binding activity specifically. $f$ The 
mutated E3 binding site was further cloned and its effect on RelB-mediated transcriptional activation was assessed by the reporter assay. ${ }^{*}(p<0.05)$ and $* *(p<0.01)$ show the significances between the two groups as indicated.

\section{Figure 4}

A
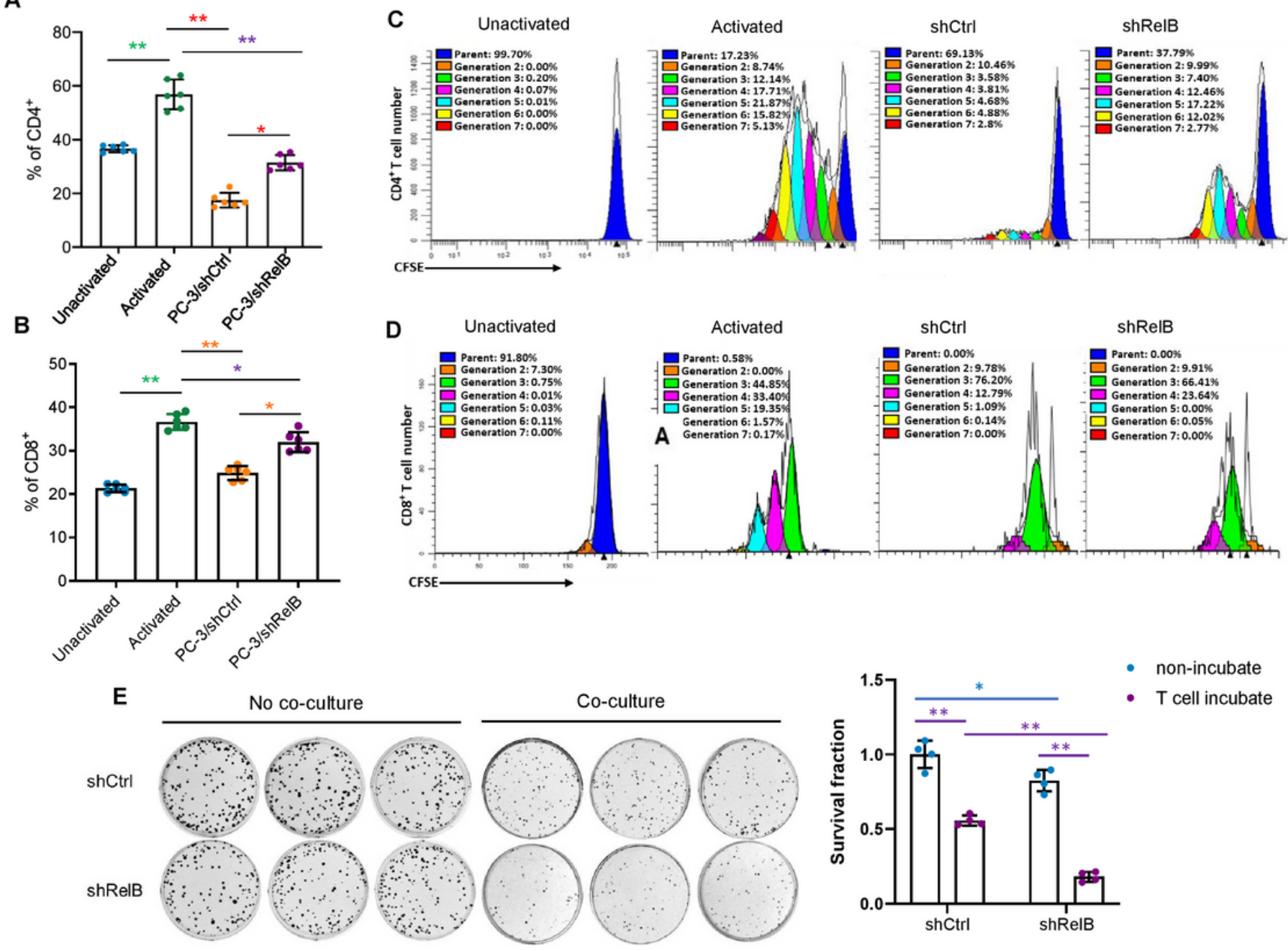

Figure 4

Silence of RelB in PC-3 cells enhances the immunities of CD4+ and CD8+ T cells. a-b PC-3 cells were cocultured with the activated T cells derived from human PBMC. CD4+ and CD8+ T cells were quantified by flow cytometry using the relative antibodies. c-d Subsequently, the proliferation of CD4+ and CD8+ T cells were analyzed by flow cytometry. The percentage of each generation (cell division) was plotted. e after co-culture with the activated T cells, the survival rate of PC-3 cells was measured using a clonogenic assay. $*(p<0.05)$ and $* *(p<0.01)$ show the significances between two groups as indicated. 
Figure 5

A

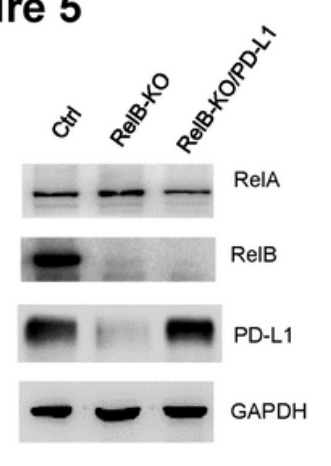

B
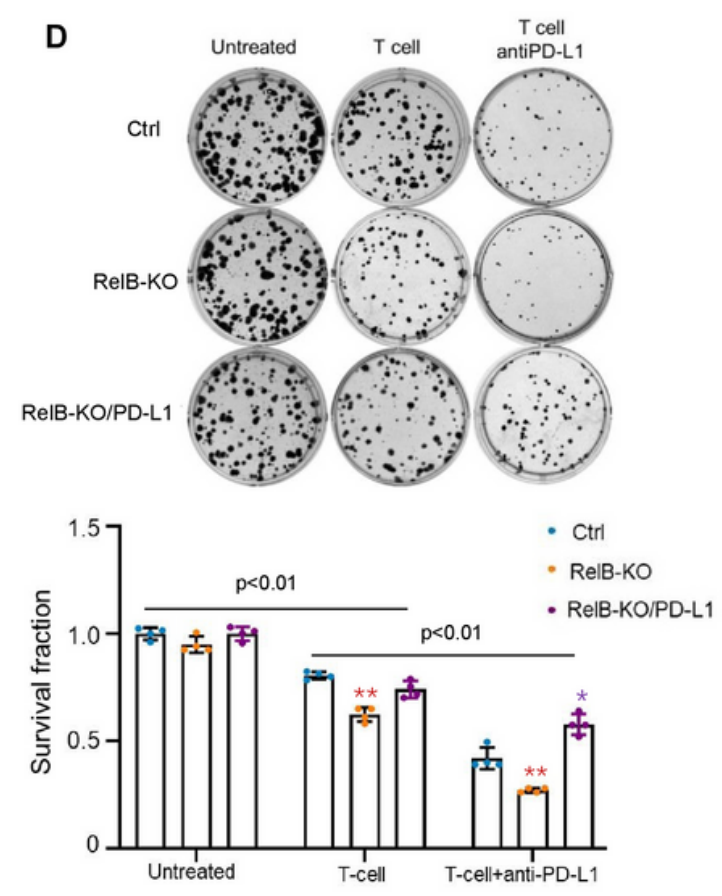

C
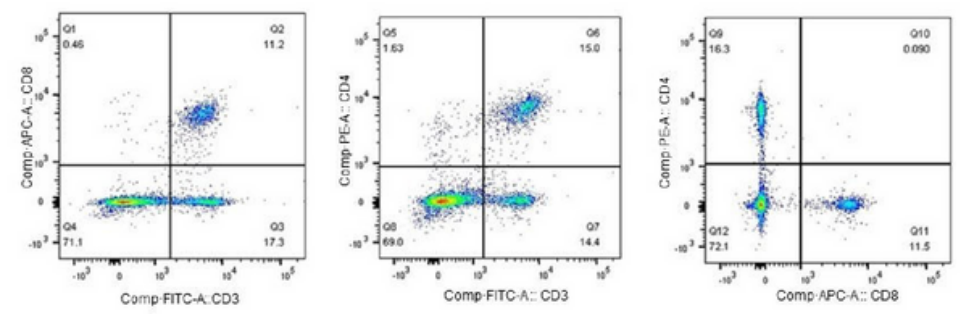

E
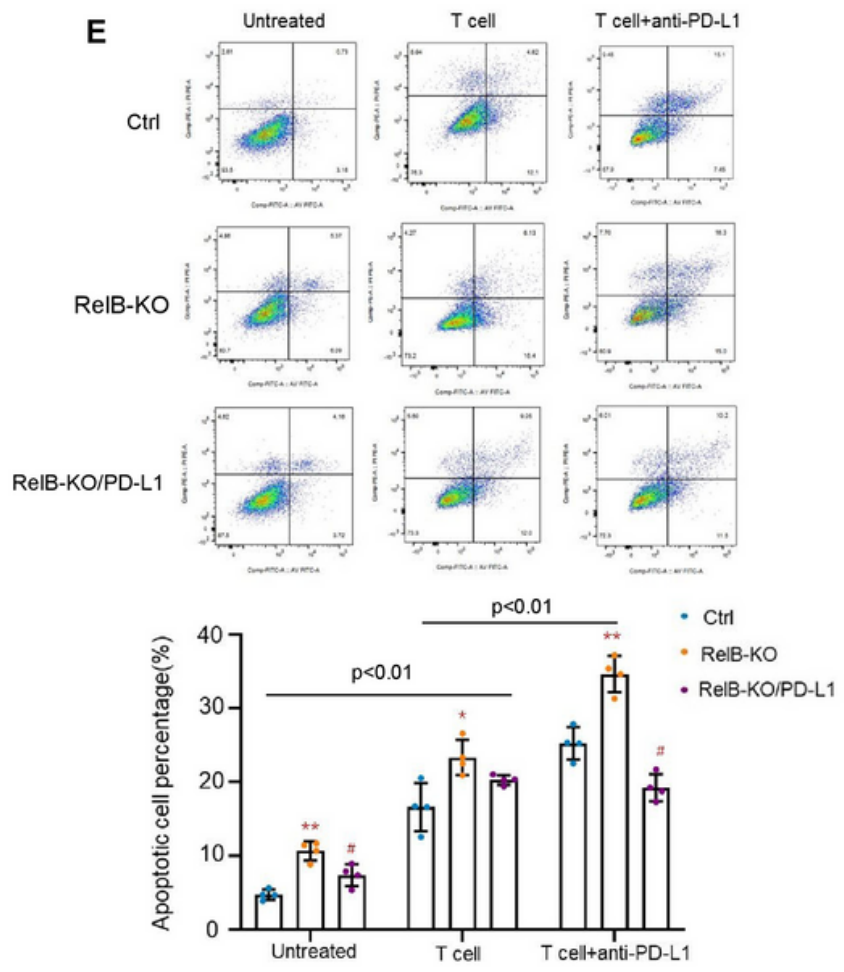

\section{Figure 5}

RelB deprivation in mouse PCa RM-1 cells restores mouse T-cell function. a RelB was knocked out from the RM-1 cells using the CRISPR/Cas9-based gene editing system and further PD-L1 was enforcedly expressed in the RelB-deprived cells to restore the immunocompromise. The cellular levels of RelA, RelB and PD-L1 proteins in these cell lines were measured by immunoblots. b Subsequently, the NF-KB binding activities in the established cell lines were quantified. ${ }^{* *}(p<0.01)$ show the significances between the two groups as indicated. $\mathrm{c} T$ cells derived from mouse spleen tissues were activated and CD4+ and CD8+ T cells were quantified by flow cytometry. $d$ RM-1 cells were treated with anti-PD-L1 mAb prior to co-culture with activated T cells, and the RM-1 cell survival was measured using a clonogenic assay. The significances between the two groups as indicated on the tap. Within same groups, $*(p<0.05), * *(p<$ 0.01 ) show the significances in RelB-KO and RelB-KO/PD-L1 cells compared to control cell. e Correspondingly, the apoptosis of RM-1 cells was further analyzed using flow cytometry. The significances between the two groups as indicated on the tap. Within same groups, $*(p<0.05), * *(p<$ $0.01)$ show the significances in RelB-KO cell vs. the control cell; and \#( $<<0.05)$ shows the significances in RelB-KO/PD-L1 cell compared to the control and RelB-KO cells. 


\section{Figure 6}
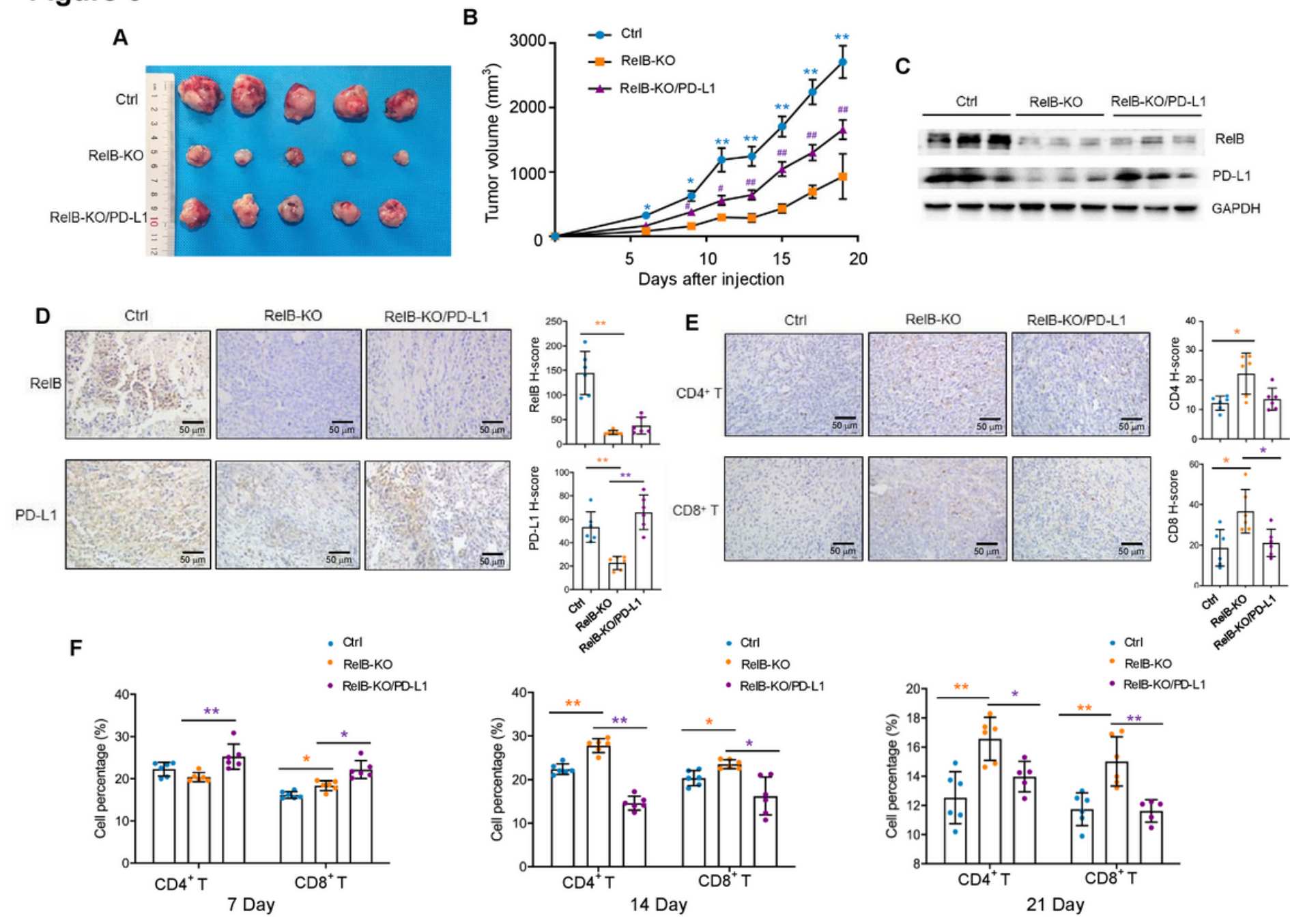

\section{Figure 6}

RelB deprivation reduces mouse xenograft tumor growth. a RM-1 (control), RM-1:RelB-KO and RM-1:RelBKO/PD-L1 cell lines were cultured and then subcutaneously injected into male C57BL/ 6 mice for tumor formation. After tumors reached the maximal volume, the mice were executed and the excised tumor tissues were photographed. $b$ Tumor volume was measured every other day and the tumor growth rate was determined and the tumor growth rate in each group was plotted. $*(p<0.05), * *(p<0.01)$ show the significances in RelB-KO cell and RelB-KO/PD-L1 compared to the control cell; and \#( $p<0.05), \# \#(p<$ 0.01 ) indicate the significances in RelB-KO/PD-L1 cell vs. RelB-KO cells. $c$ The expression levels of RelB and PD-L1 in the tumor tissues were measured by immunoblots. d-e The levels of RelB, PD-L1, CD4 and CD8 proteins in the tumor tissues were quantified by $\mathrm{IHC}$ and the relative $\mathrm{H}$-scores were plotted. $*(\mathrm{p}<$ $0.05), * *(p<0.01)$ show the significances between two groups as indicated. $f$ Blood samples were collected from mice on different days after cell injection as indicated to analyze the percentages of CD4+ and CD $8+T$ cells by flow cytometry. ${ }^{*}(p<0.05),{ }^{* *}(p<0.01)$ show the significances between two groups as indicated. 
Figure 7
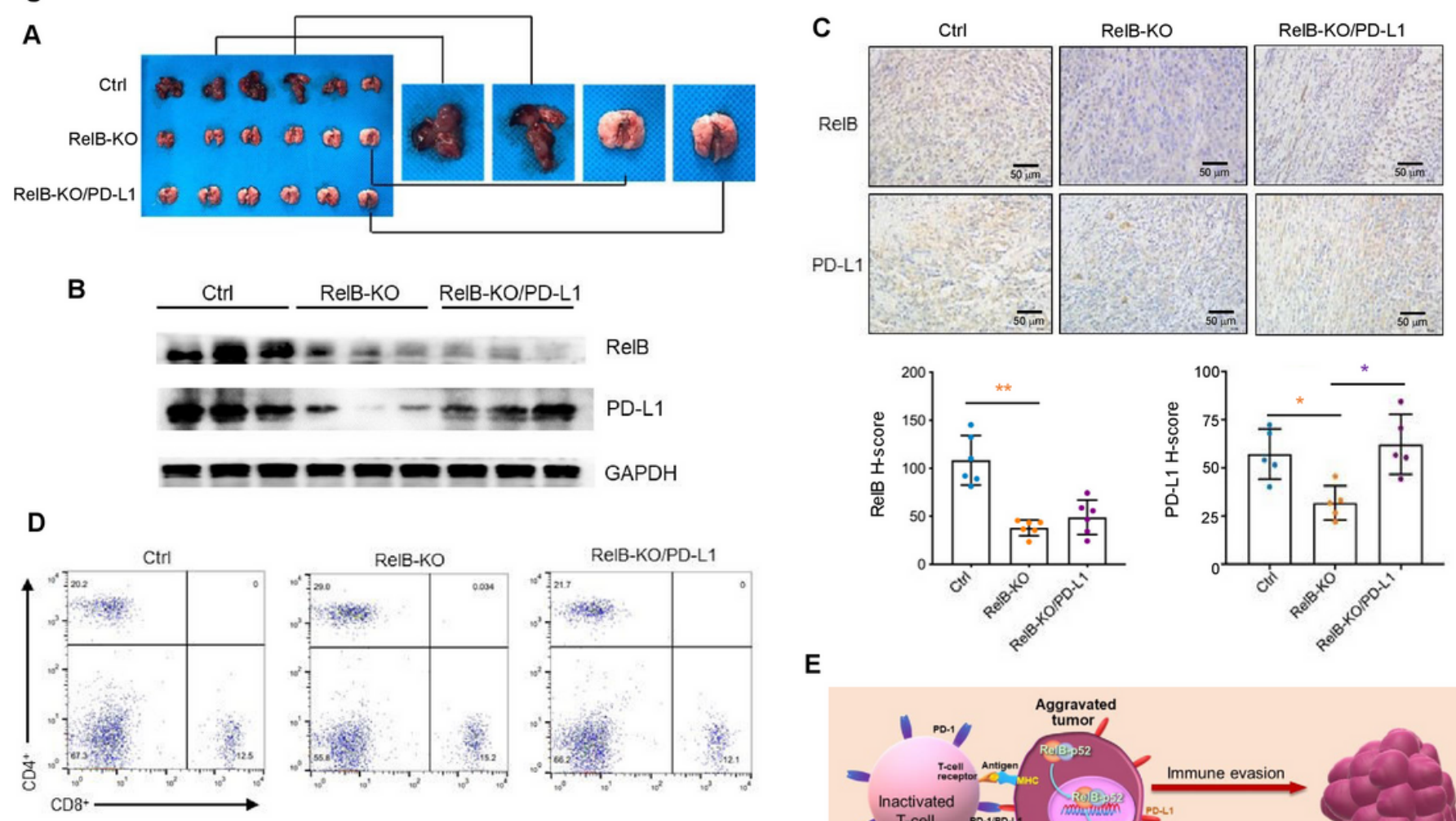

E

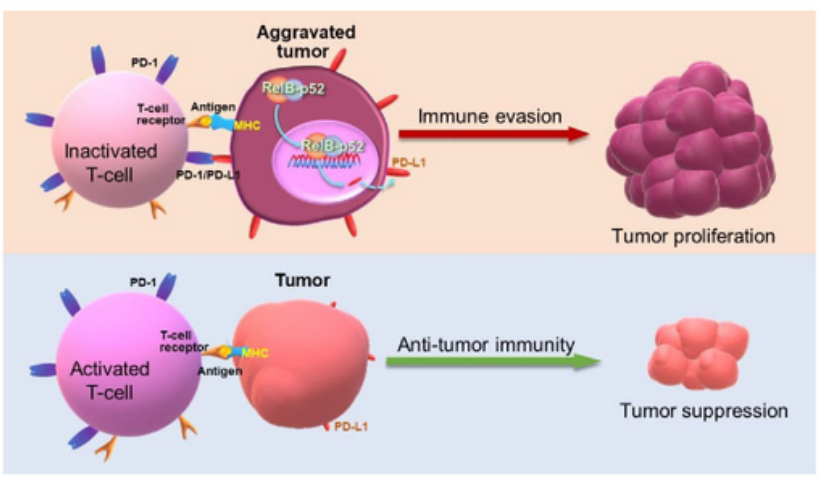

\section{Figure 7}

RelB deprivation decreases mouse xenograft tumor lung metastasis. a RM-1 and its generated cell lines were intravenously injected into male C57BL/6 mice. Lung tissues with tumors in the control group and without tumors in the RelB-KO groups were excised and examined. b The expression levels of RelB and PD-L1 in the excised tissues were measured by immunoblots. GAPDH-normalized protein levels were plotted. c Additionally, the expression of RelB and PD-L1 was further quantified by IHC and the relative $\mathrm{H}$ scores were plotted. $d$ The percentages of CD4+ and CD8+ T cells derived from mice blood samples were analyzed by flow cytometry. ${ }^{*}(p<0.05),{ }^{*}(p<0.01)$ show the significances between two groups as indicated. e Depiction of the suggested mechanism underlying RelB-mediated immune evasion of PCa cells.

\section{Supplementary Files}

This is a list of supplementary files associated with this preprint. Click to download. 
- Supplementarymaterials.pdf 